\title{
Generalized parton distributions of the nucleon from twisted mass QCD
}

\author{
Constantia Alexandrou ${ }^{(a, b)}$, Martha Constantinou ${ }^{(a)}$, Tomasz Korzec ${ }^{* i(a)}$ \\ (a) Department of Physics, University of Cyprus, P.O. Box 20537, 1678 Nicosia, Cyprus \\ (b) Computation-based Science and Technology Research Center, The Cyprus Institute, 15 \\ Kypranoros Str., 1645 Nicosia, Cyprus \\ E-mail: alexand@ucy.ac.cy, marthaceucy.ac.cy, korzec@ucy.ac.cy
}

\section{Mariane Brinet, Jaume Carbonell, Pierre-Antoine Harraud}

Laboratoire de Physique Subatomique et Cosmologie, 53 avenue des Martyrs, 38026 Grenoble,

France

E-mail: marianedlpsc.in2p3.fr, carbonelelpsc.in2p3.fr

harraudelpsc.in $2 \mathrm{p} 3 . \mathrm{fr}$

\section{Rémi Baron}

CEA, Centre de Saclay, IRFU/Service de Physique Nucléaire, F-91191 Gif-sur-Yvette, France

E-mail: remi.barondcea.fr

\section{Karl Jansen, Dru Renner}

NIC / DESY Zeuthen, Platanenallee 6, D-15738 Zeuthen

E-mail: Karl.Jansen@desy.de, dru.renner@desy.de

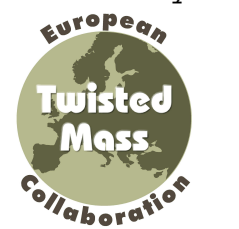

We calculate the generalized form factors associated with the one derivative axial and vector operators in two flavor Wilson-twisted-mass QCD. At zero momentum transfer these reduce to the first moments of parton distributions, the momentum fraction $\langle x\rangle$ and the spin fraction $\langle\Delta x\rangle$. We briefly describe our non-perturbative renormalization procedure, which is done in the RI'MOM scheme. The calculation of physical observables is carried out at different pion masses, down to approximately $300 \mathrm{MeV}$ at a lattice spacing of about $0.089 \mathrm{fm}$ and in a spatial volume of about $2.1 \mathrm{fm}$.

The XXVII International Symposium on Lattice Field Theory

July 26-31, 2009

Peking University, Beijing, China

\footnotetext{
* Speaker.

${ }^{\dagger}$ Current address: Institut für Physik, Humboldt Universität zu Berlin, Newtonstrasse 15, 12489 Berlin, Germany.
} 


\section{Introduction}

Lattice QCD calculations of observables related to the structure of baryons are becoming increasingly relevant since direct connection to experiment can now be made. This is due to the fact that systematic uncertainties caused by a finite volume, a finite lattice cut-off and unphysically high pion masses are becoming better controlled. Nowadays a number of major collaborations are producing results on nucleon form factors and the first moments of parton distributions closer to the physical regime both in terms of the pion mass and the lattice spacing [1, 2, 3, 4, 5]. Generalized parton distributions (GPDs) encode important information related to baryon structure and can be studied in a systematic way. While experiments are able to measure convolutions of GPDs, lattice QCD allows us to extract generalized form factors of operators like

$$
\mathscr{O}_{V^{a}}^{\mu_{1} \ldots \mu_{n}}=\bar{\psi} D^{\left\{\mu_{1}\right.} \ldots D^{\mu_{n-1}} \gamma^{\left.\mu_{n}\right\}} \frac{\tau^{a}}{2} \psi, \quad \mathscr{O}_{A^{a}}^{\mu_{1} \ldots \mu_{n}}=\bar{\psi} D^{\left\{\mu_{1}\right.} \cdots D^{\mu_{n-1}} \gamma^{\left.\mu_{n}\right\}} \gamma^{5} \frac{\tau^{a}}{2} \psi
$$

from which GPDs can be reconstructed via inverse Mellin transforms. The special case of $n=0$, for which eq. (1.1) reduces to the vector and axial current with the associated Dirac, Pauli and axial form factors, is treated separately and results are outlined in ref. [6]. In this work we concentrate on the $n=1$ case for which the nucleon matrix elements can be decomposed in terms of the generalized form factors $A_{20}\left(q^{2}\right), B_{20}\left(q^{2}\right), C_{20}\left(q^{2}\right)$ and $\tilde{A}_{20}\left(q^{2}\right), \tilde{B}_{20}\left(q^{2}\right)$, as follows,

$$
\begin{aligned}
& \left\langle N\left(p_{f}, s_{f}\right)\left|\mathscr{O}_{V}^{\mu v}\right| N\left(p_{i}, s_{i}\right)\right\rangle=\bar{v}\left(p_{f}, s_{f}\right)\left[A_{20} \gamma^{\{\mu} q^{v\}}+B_{20} \frac{i \sigma^{\{\mu \alpha} q_{\alpha} q^{v\}}}{2 m}+C_{20} \frac{1}{m} q^{\{\mu} q^{v\}}\right] v\left(p_{i}, s_{i}\right), \\
& \left\langle N\left(p_{f}, s_{f}\right)\left|\mathscr{O}_{A}^{\mu v}\right| N\left(p_{i}, s_{i}\right)\right\rangle=\bar{v}\left(p_{f}, s_{f}\right)\left[\tilde{A}_{20} \gamma^{\{\mu} q^{v\}} \gamma^{5}+\tilde{B}_{20} \frac{q^{\{\mu} q^{\nu\}}}{2 m} \gamma^{5}\right] v\left(p_{i}, s_{i}\right) .
\end{aligned}
$$

Here $q=p_{f}-p_{i}$ is the momentum transfer, $v$ is a Dirac spinor and the brackets $\{\cdot\}$ stand for symmetrization over all uncontracted indices and subtraction of the traces.

\section{Extraction of the generalized form factors}

We work with two degenerate dynamical flavors of twisted mass Wilson fermions and with a tree level improved Symanzik gauge action. For simulation details the reader is referred to ref. [7].

Methods developed for the extraction of ordinary nucleon form factors [ [ carry over almost unaltered to the present case. We calculate the following two- and three point functions

$$
\begin{aligned}
G(\vec{q}, t) & =\sum_{\vec{x}_{f}} e^{-i \vec{x}_{f} \cdot \vec{q}} \Gamma_{\beta \alpha}^{0}\left\langle J_{\alpha}\left(t_{f}, \vec{x}_{f}\right) \bar{J}_{\beta}(0)\right\rangle, \\
G_{\mu v}\left(\Gamma^{\rho}, \vec{q}, t\right) & =\sum_{\vec{x}, \vec{x}_{f}} e^{i \vec{x} \cdot \vec{q}} \Gamma_{\beta \alpha}^{\rho}\left\langle J_{\alpha}\left(t_{f}, \vec{x}_{f}\right) \mathscr{O}_{\mu v}(t, \vec{x}) \bar{J}_{\beta}(0)\right\rangle,
\end{aligned}
$$

and form the ratios

$$
R_{\mu v}(\Gamma, \vec{q}, t)=\frac{G_{\mu v}(\Gamma, \vec{q}, t)}{G\left(\overrightarrow{0}, t_{f}\right)} \sqrt{\frac{G\left(\vec{q}, t_{f}-t\right) G(\overrightarrow{0}, t) G\left(\overrightarrow{0}, t_{f}\right)}{G\left(\overrightarrow{0}, t_{f}-t\right) G(\vec{q}, t) G\left(\vec{q}, t_{f}\right)}} .
$$


Like in the preceding calculations of masses and form factors, the proton interpolating field $J(x)=$ $\varepsilon^{a b c}\left[u^{a \top}(x) \mathscr{C} \gamma_{5} d^{b}(x)\right] u^{c}(x)$, is constructed from smeared quark fields (here and later $u$ and $d$ denote the quark fields in the physical basis). Gaussian smearing of the fermions and APE smearing of gauge-fields entering the smearing function are employed with the parameters tuned as in ref. [8]. The sequential inversion "through the sink" technique that we use here forces us to fix the source-sink separation $t_{f}-t_{i}=12 a$, the smearing parameters and the matrix $\Gamma$ at the beginning of the calculation. The advantage is that any local or non-local bilinear operator carrying an arbitrary lattice momentum can be inserted without the requirement of further inversions. In fact the four choices $\Gamma^{0}=1 / 4\left[\mathbb{1}+\gamma_{0}\right], \Gamma^{k}=i \Gamma^{0} \gamma_{5} \gamma_{k}$ are identical to those used in the calculation of Sachs and axial form factors. For sufficiently large separations $t_{f}-t$ and $t-t_{i}$ the ratio of eq. 2.3 exhibits a plateau and we denote the fitted plateau value by $\Pi_{\mu v}(\Gamma, \vec{q})$. From renormalized plateau values $\Pi_{R}=Z \Pi$ the generalized form factors can be extracted. All values of $\vec{q}$ resulting in the same $q^{2}$, the four choices of $\Gamma$ and the ten orientations $\mu v$ of the operator lead to an over-constrained system of equations which is solved in the least-squares sense via a singular value decomposition of the coefficient matrix. The coefficients follow from the matrix-element decomposition given in eq. (1.2) and may depend on the energy and mass of the nucleon as well as on the initial spatial momentum $\vec{p}_{i}=-\vec{q}$. It turns out that both the operators with $\mu=v$ and $\mu \neq v$ are necessary to obtain all three vector form factors. Since those two classes of operators on a hypercubic lattice renormalize differently from each other [9], renormalization has to be carried out already on the level of the ratios. Fig. 1 1 shows some of the plateaus we observe in the ratios.
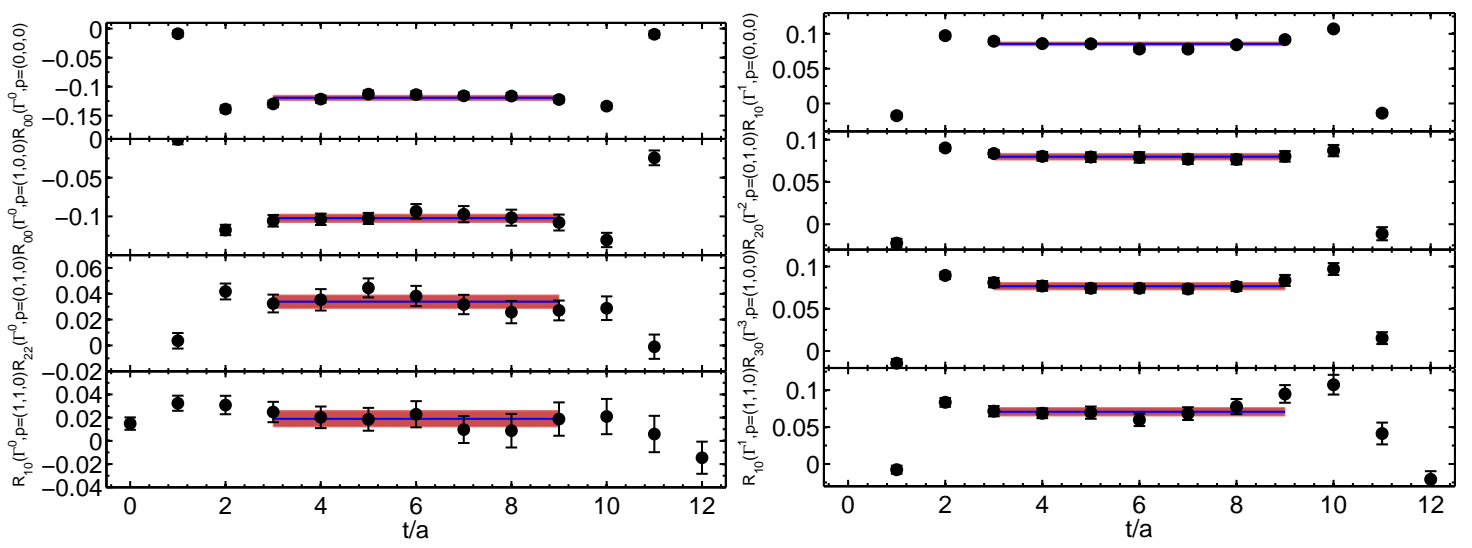

Figure 1: Ratios for the one derivative vector (left) and axial (right) operator for a few exemplary choices of the momentum. The solid lines with the bands indicate the fitted plateau values with their jackknife errors.

\section{Non-perturbative renormalization}

The operators defined in eq. (1.1) require renormalization. We compute the necessary renormalization constants in the RI'-MOM scheme at different renormalization scales non-perturbatively and use perturbation theory to translate them to the popular $\overline{M S}$-scheme at $2 \mathrm{GeV}$. A similar procedure has been employed by the authors of ref. [10]. In addition, we subtract perturbatively to one loop the $\mathscr{O}\left(a^{2}\right)$ contributions as described below. For the present calculation we will need the one 
derivative vector operator with $\mu=v\left(Z_{V D 1}\right)$, the one derivative vector operator with $\mu \neq v\left(Z_{V D 2}\right)$ and the one derivative axial operator with $\mu \neq v\left(Z_{A D 2}\right)$.

The operators can all be written in the form

$$
\mathscr{O}=\sum_{z^{\prime}} \bar{u}(z) \mathscr{J}\left(z, z^{\prime}\right) d\left(z^{\prime}\right),
$$

where $\mathscr{J}$ determines the operator, e.g. $\mathscr{J}\left(z, z^{\prime}\right)=\delta_{z, z^{\prime}} \gamma_{\mu}$ would correspond to the local vector current. For each operator we define a bare vertex function $(12 \times 12$ matrix $)$ given by

$$
G(p)=\frac{a^{12}}{V} \sum_{x, y, z, z^{\prime}} e^{-i p(x-y)}\left\langle u(x) \bar{u}(z) \mathscr{J}\left(z, z^{\prime}\right) d\left(z^{\prime}\right) \bar{d}(y)\right\rangle,
$$

where $p$ is a momentum allowed by the boundary conditions, and the gauge average is performed over gauge-fixed configurations. We fix to Landau gauge using a stochastic over-relaxation algorithm [11]. In this work, we do not address questions related to the Gribov ambiguity.

The propagator in momentum space is defined by

$$
S^{u}(p)=\frac{a^{8}}{V} \sum_{x, y} e^{-i p(x-y)}\langle u(x) \bar{u}(y)\rangle, \quad S^{d}(p)=\frac{a^{8}}{V} \sum_{x, y} e^{-i p(x-y)}\langle d(x) \bar{d}(y)\rangle,
$$

and an amputated vertex function is given by

$$
\Gamma(p)=\left(S^{u}(p)\right)^{-1} G(p)\left(S^{d}(p)\right)^{-1} .
$$

The corresponding renormalized quantities are

$$
S_{R}(p)=Z_{q} S(p), \quad \Gamma_{R}(p)=Z_{q}^{-1} Z_{\mathscr{O}} \Gamma(p),
$$

with Z-factors determined by the renormalization conditions of the RI'-MOM scheme

$$
\left.\frac{1}{12} \operatorname{tr}\left[S_{R}^{-1}(p) S^{(0)}(p)\right]\right|_{p^{2}=\mu^{2}}=1 \quad \text { and }\left.\quad \frac{1}{12} \operatorname{tr}\left[\Gamma_{R}(p) \Gamma^{(0)-1}(p)\right]\right|_{p^{2}=\mu^{2}}=1
$$

where $\mu$ is the renormalization scale and $S^{(0)}$ and $\Gamma^{(0)}$ are tree level expressions for $S$ and $\Gamma$. These are imposed in the massless theory, i.e. at critical mass and vanishing twisted mass. We evaluate eq. (3.2) and eq. (3.3) for each momentum separately employing Fourier sources. Alternatively one could exploit translation invariance to shift the operator position in eq. (3.2) to position $z=0$ in each term. This would allow for an evaluation of the vertex function with all possible momenta at the cost of one set of inversions per configuration, but would lead to larger statistical errors. This second method has been carried out for local bilinears [12] and one of the 1-derivative operators [13] on the same configurations, leading to compatible results.

Chiral extrapolations are necessary to obtain the renormalization factors in the chiral limit. As can be seen in Fig. 2 for six different renormalization scales, the pion mass dependence is very mild and a linear extrapolation suffices. We use a 1-loop expression [10] to convert our results to the $\overline{M S}$-scheme and a 2-loop formula [10, 14] to evolve the renormalization scale down to $\mu=2 \mathrm{GeV}$. If a "renormalization window" exists where $\Lambda_{Q C D}^{2}<<\mu^{2}<<1 / a^{2}$ holds, we expect a plateau. In reality the upper inequality is not satisfied and we see pronounced lattice artifacts in our results. 

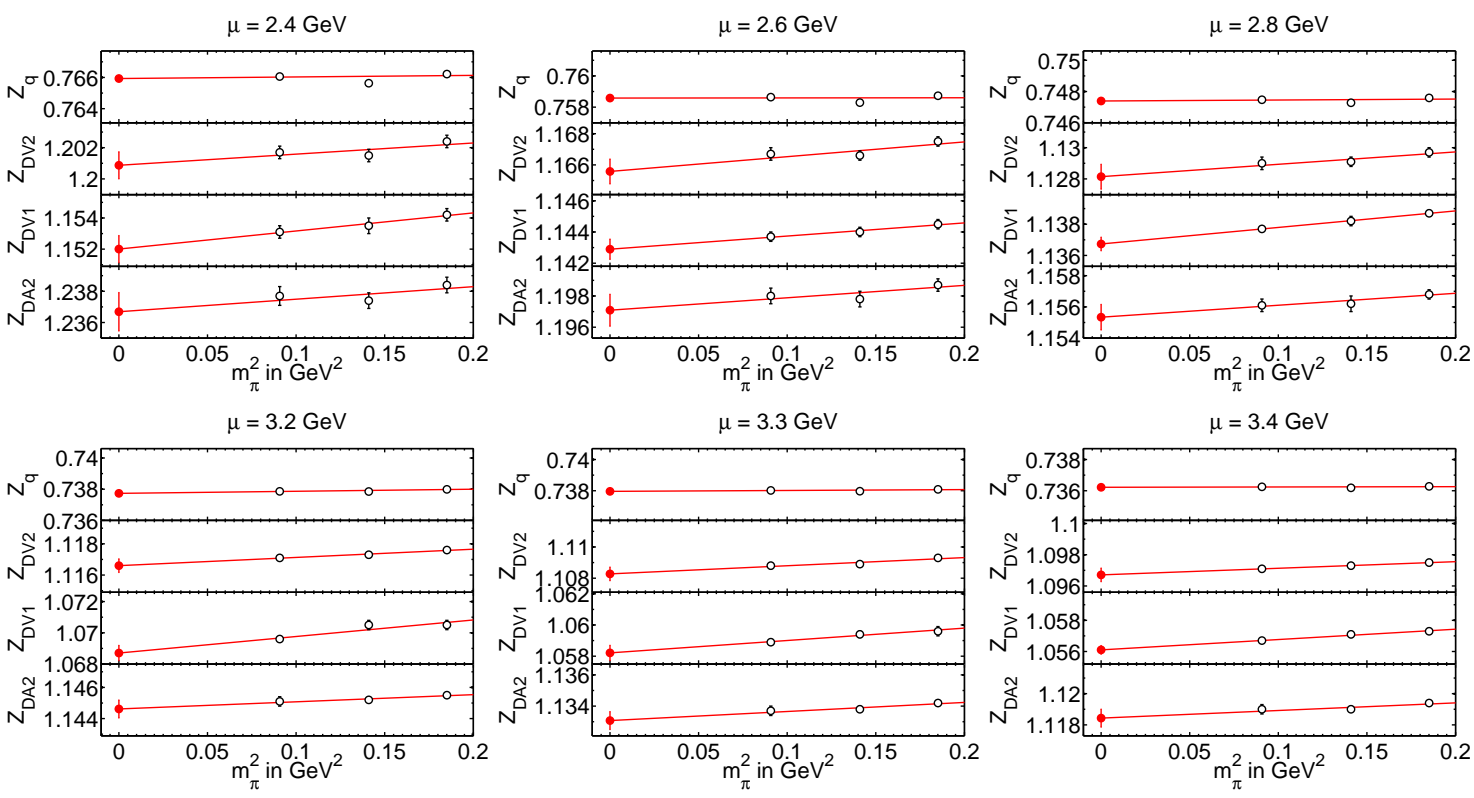

Figure 2: Chiral extrapolations of the Z-factors for six different renormalization scales.

Fortunately recent perturbative calculations [15] allow us to subtract the perturbative $O\left(a^{2}\right)$ lattice artifacts to one loop, which alleviates the problem. To remove the remaining $O\left(g^{4} a^{2} p^{2}\right)$ artifacts we extrapolate linearly to $a^{2} p^{2}=0$. The statistical errors are negligible compared to the systematic ones. The whole procedure is demonstrated in Fig. B. Our preliminary results for the $Z$-factors in the $\overline{M S}$-scheme at $2 \mathrm{GeV}$ are

$$
Z_{D V 1}=1.17(2), \quad Z_{D V 2}=1.15(9), \quad Z_{D A 2}=1.19(1) .
$$

The error is the systematic error due to the extrapolation, namely the difference between using all points or just the higher four ones. Statistical errors are at least one order of magnitude smaller.

\section{Results}

In Fig. $\bigoplus$ we show our results for the renormalized generalized form factors of the one derivative vector operator. Qualitative features, like the ordering of $A_{20}, B_{20}$ and $C_{20}$ or the momentum dependence are in agreement with the results of ref. [1]. Quantitatively however our results are larger and closer to those obtained by the QCDSF collaboration [3]. Since both us and the QCDSF collaboration use a non-perturbative renormalization this perhaps may be attributed to the different renormalization procedures (perturbative versus non-perturbative). Our values for $\langle x\rangle_{u-d}=A_{20}(0)$ are compatible with results by several other groups and, at the currently available pion masses, deviate from the phenomenological value $\langle x\rangle \approx 0.16$. In fig. 5 we summarize our results for the axial one derivative operator.

Extending the present calculation to the other available twisted mass fermion ensembles will be crucial to understand the considerable deviations from experiment that are currently observed in all lattice calculations of $\langle x\rangle$ and $\langle\Delta x\rangle$. 


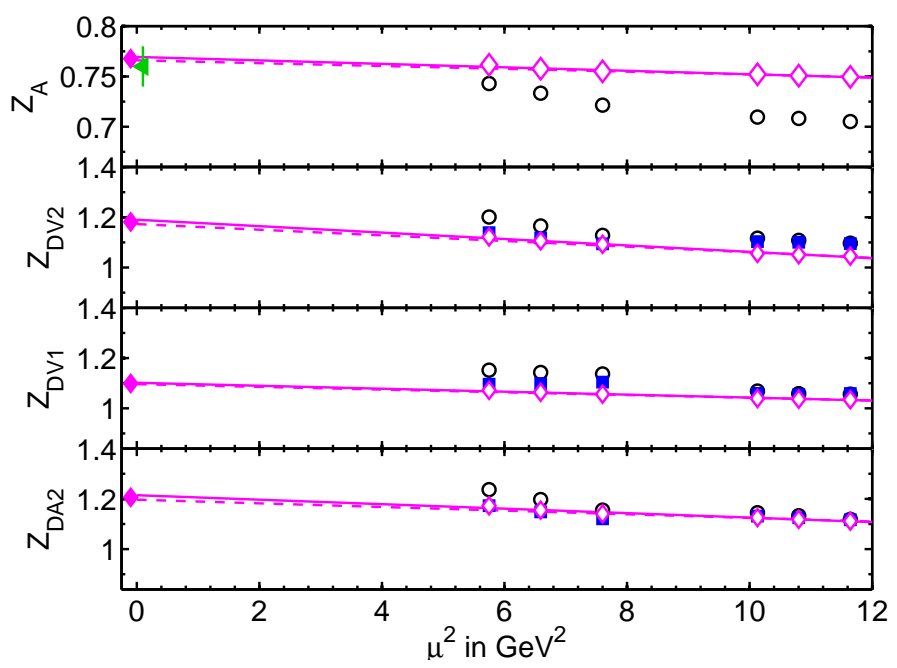

Figure 3: Renormalization factors in the RI'-MOM scheme at renormalization scale $\mu$ (circles). Results translated to the $\overline{M S}$-scheme at renormalization scale $2 \mathrm{GeV}$ (filled squares) and results with perturbatively subtracted one loop $O\left(a^{2}\right)$ artifacts (diamonds). The lines show extrapolations to $a^{2} p^{2}=0$ using all (solid) or just the last four points (dashed). The filled triangle is $Z_{A}$ from ref. [12].
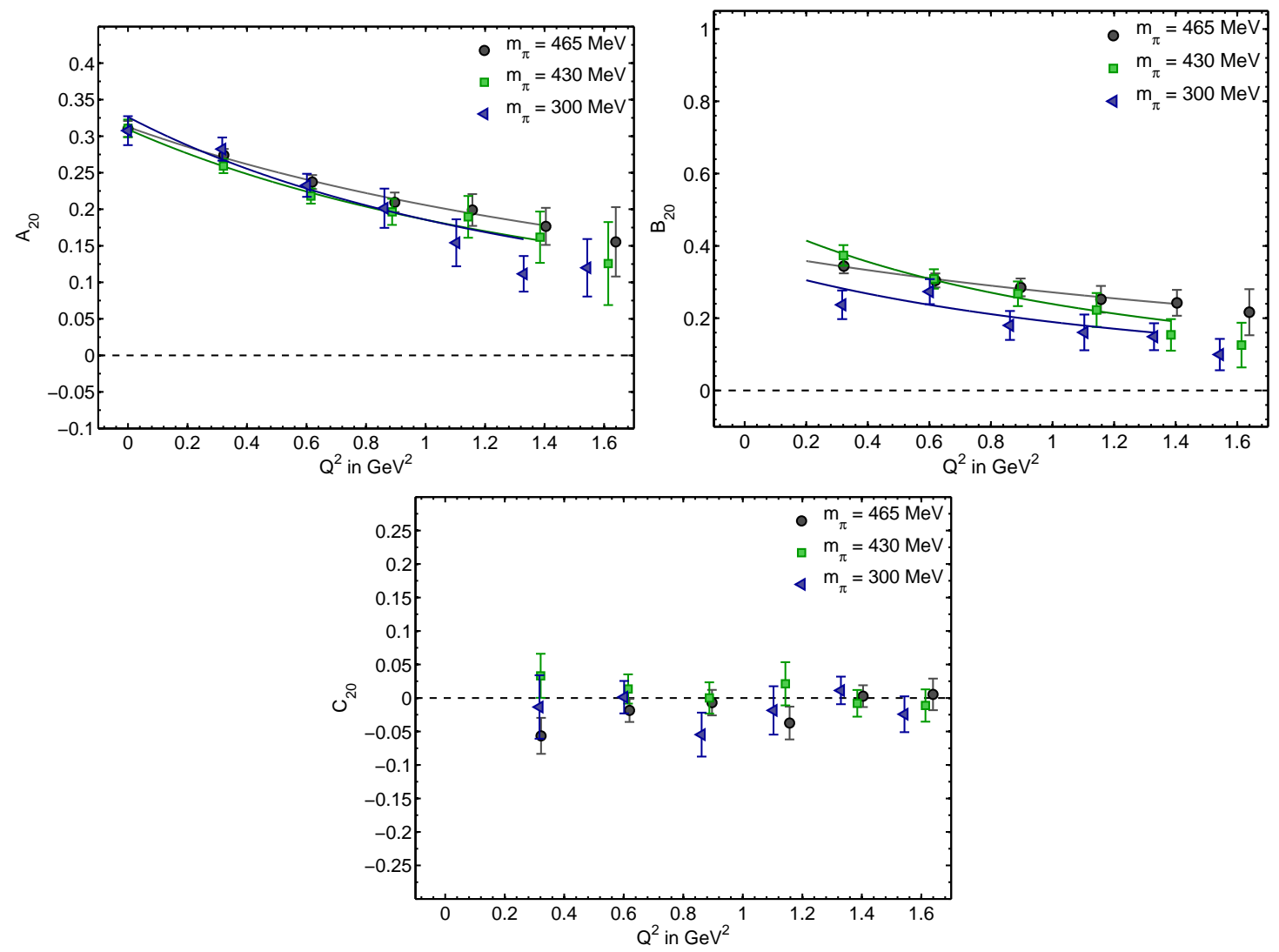

Figure 4: The figure shows our results for the generalized form factors $A_{20}, B_{20}$ and $C_{20}$ calculated at different pion masses. The solid lines are dipole fits included here to guide the eye. 

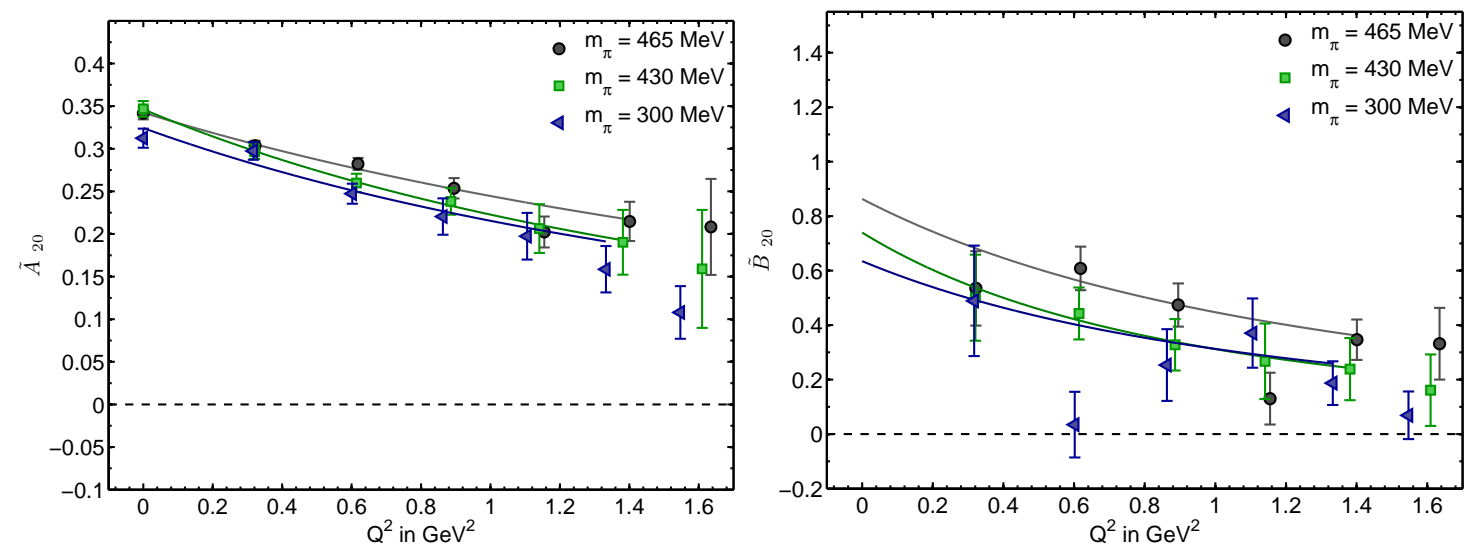

Figure 5: The figure shows the generalized form factors $\tilde{A}_{20}$ and $\tilde{B}_{20}$ calculated at different pion masses.

\section{Acknowledgments}

C.A., M.C. and T.K. would like to thank Haralambos Panagopoulos for fruitful discussions. This work was partly supported by funding received from the Cyprus Research Promotion Founda-

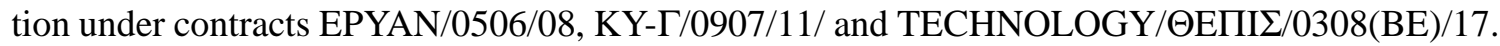
HPC resources from GENCI (DRIS and CINES) Grant 2009-052271 and CCIN2P3 (Lyon) were used.

\section{References}

[1] LHPC, P. Hagler et al., Phys. Rev. D77 (2008) 094502, arXiv:0705.4295.

[2] S.N. Syritsyn et al., (2009), arXiv:0907.4194.

[3] QCDSF-UKQCD, D. Brommel et al., PoS LAT2007 (2007) 158, arXiv:0710.1534.

[4] ETMC, C. Alexandrou et al., (2008), arXiv:0811.0724.

[5] T. Yamazaki et al., Phys. Rev. D79 (2009) 114505, arXiv:0904.2039.

[6] ETMC, C. Alexandrou et al., PoS LAT2009 (2009) 145.

[7] ETMC, P. Boucaud et al., Comput. Phys. Commun. 179 (2008) 695, arXiv:0803.0224.

[8] ETMC, C. Alexandrou et al., Phys. Rev. D78 (2008) 014509, arXiv:0803.3190.

[9] M. Gockeler et al., Phys. Rev. D54 (1996) 5705, hep-lat/9602029.

[10] M. Gockeler et al., Nucl. Phys. B544 (1999) 699, hep-lat/9807044.

[11] P. de Forcrand, Nucl. Phys. Proc. Suppl. 9 (1989) 516.

[12] P. Dimopoulos et al., PoS LAT2007 (2007) 241, arXiv:0710.0975.

[13] Z. Liu and O. Pene, private communication .

[14] E.G. Floratos, D.A. Ross and C.T. Sachrajda, Nucl. Phys. B129 (1977) 66.

[15] M. Constantinou, H. Panagopoulos and F. Stylianou, PoS LAT2009 (2009) 205. 